\title{
Cytoskeleton changes of airway smooth muscle cells in juvenile rats with airway remodeling in asthma and the RhoA/ROCK signaling pathway mechanism
}

\author{
B. Wei', Y.X. Shang ${ }^{2}$, M. Li ${ }^{2}$, J. Jiang ${ }^{1}$ and H. Zhang ${ }^{2}$ \\ 'Department of Pediatrics, \\ General Hospital of Shenyang Military Area Command, Shenyang, China \\ ${ }^{2}$ Department of Pediatrics, Shengjing Hospital of China Medical University, \\ China Medical University, Shenyang, China \\ Corresponding author: B. Wei \\ E-mail: bwyxcn@163.com
}

Genet. Mol. Res. 13 (1): 559-569 (2014)

Received August 29, 2013

Accepted November 7, 2013

Published January 22, 2014

DOI http://dx.doi.org/10.4238/2014.January.22.2

\begin{abstract}
This article aimed to investigate changes in the cytoskeleton of airway smooth muscle cells (ASMCs) in juvenile rats with airway remodeling in asthma. We further investigated the involvement of the RhoA/ROCK signaling pathway mechanism. Rat models of airway remodeling in asthma were established by antigen sensitization with ovalbumin for 2, 4, 6, and 8 weeks. The control group was treated with normal saline instead of ovalbumin. In the intervention group, after 8 weeks of culture, ASMCs were treated with the ROCKspecific inhibitor Y-27632. Immunofluorescence, real-time polymerase chain reaction, and Western blot analyses were used to observe changes in the cytoskeleton (F-actin and $\alpha$-tubulin) of ASMCs and expressions of RhoA and ROCK. The asthmatic groups had significantly higher average gray values of F-actin in ASMCs compared to the control group $(\mathrm{P}<0.01)$, and these values for the intervention group were
\end{abstract}


significantly lower than those of the 8-week asthmatic group $(\mathrm{P}<0.05)$. Expression levels of the $\alpha$-tubulin protein in the asthmatic groups were all significantly higher than those of the control group $(\mathrm{P}<0.01)$, and the levels in the intervention group were significantly reduced $(\mathrm{P}<0.05)$. Expressions of RhoA and ROCK mRNA and proteins in all asthmatic groups were significantly higher than those of the control group $(\mathrm{P}<$ $0.01)$. Together, these results demonstrate substantial changes of the ASMC cytoskeleton and abnormal expressions of RhoA and ROCK mRNA and proteins in juvenile rats with airway remodeling in asthma.

Key words: Asthma; Airway remodeling; Cytoskeleton; RhoA/ROCK; Rat

\section{INTRODUCTION}

The cytoskeleton is a three-dimensional grid structure composed of a variety of structural and contractile proteins in a particular pattern. It is an important and substantial foundation for maintaining specific cell morphologies and exercising systolic functions. The cytoskeleton exists in three main forms including microfilaments, microtubules, and intermediate filaments. Recent studies have revealed that the RhoA/ROCK signaling pathway in the small $\mathrm{G}$ protein family can regulate the reconstruction of microfilaments in smooth muscle cells by mediating protein phosphorylation, thus affecting the biological behavior of cells (Fukata et al., 2001; Stamenović, 2008). Therefore, investigating the regulatory mechanism of the RhoA/ROCK signaling pathway involved in the reconstruction of airway smooth muscle cells (ASMCs) in airway remodeling in asthma, and its pathological physiological significance, can contribute to greater understanding of the pathogenesis of this disease. However, few studies of this nature have been conducted to date.

In this study, ASMCs of Sprague-Dawley (SD) rats were cultured and treated with a ROCK inhibitor. Immunofluorescence, real-time quantitative polymerase chain reaction (qPCR), and Western blot methods were used to observe expressions of F-actin, $\alpha$-tubulin, RhoA, and ROCK. Changes in the cytoskeleton of ASMCs and the RhoA/ROCK signaling pathway mechanism in airway remodeling in asthma are discussed.

\section{MATERIAL AND METHODS}

\section{Experimental animals and materials}

Specific-pathogen-free juvenile female SD rats $(60-80 \mathrm{~g})$ were obtained from the Experimental Animal Center of China Medical University. RNAiso ${ }^{\mathrm{TM}}$ Plus and the qPCR kit were obtained from TaKaRa Co., Ltd. (Mountain View, CA, USA). RhoA and ROCK primers and the SeeBlue ${ }^{\mathrm{TM}}$ protein marker were purchased from the Invitrogen Biotechnology Co. Ltd. (Grand Island, NY, USA). Dulbecco's modified Eagle's medium/Ham's F-12 (DMEM/F12) and fetal bovine serum (FBS) were purchased from HyClone Laboratories, Inc. (Logan, UT, USA). Collagenase type IV was obtained from Gibco Inc. (USA). Phalloidin-fluorescein isothiocyante (FITC), 4',6-diamidino-2-phenylindole (DAPI) and Y-27632 were purchased from 
Sigma-Aldrich, Inc. (St. Louis, MO, USA). Rabbit anti-rat $\alpha$-tubulin antibody was obtained from Santa Cruz Biotechnology, Inc. (Santa Cruz, CA, USA), and the Total Protein Extraction Kit was purchased from Nanjing KGI Biological Technology Development Co., Ltd. (China).

\section{Establishment of rat models of airway remodeling in asthma}

Rats were injected with $1 \mathrm{~mL}$ antigen sensitization liquid (100 mg ovalbumin, $100 \mathrm{mg}$ aluminum hydroxide, and $5 \times 10^{9}$ inactivated Bordetella pertussis) on the 1st and 8th day of the experiment. Rats injected with an equal volume of saline were used as controls. As of the 15 th day of the experiment, rats were excited by ultrasonic atomizing inhalation with $1 \%$ ovalbumin for 30 min 3 times per week. Rats were divided into control group, 2-week asthmatic group, 4-week asthmatic group, 6-week asthmatic group, 8-week asthmatic group, and the intervention group. The excitation was performed for $2,4,6$, and 8 weeks in each asthmatic group. The control group was treated with normal saline instead of ovalbumin for 2 weeks. After 8 weeks of culture, ASMCs in rats of the intervention group were treated with $10 \mu \mathrm{M}$ ROCK-specific inhibitor, Y-27632, followed by $24 \mathrm{~h}$ of continued culture.

\section{Primary culture of rat ASMCs}

After routine disinfection and anesthesia, rats were executed by drawing left ventricular blood. Under sterile conditions, the trachea was isolated, followed by careful removal of outer and internal membranes. The tracheal smooth muscle was removed, cut into pieces, and $0.1 \%$ trypsin was added, followed by oscillation in a water bath at $37^{\circ} \mathrm{C}$ for $10 \mathrm{~min}$. After centrifugation at $1000 \mathrm{rpm}$ for $5 \mathrm{~min}$, the supernatant was removed. Then, $0.1 \%$ collagenase type IV was added, followed by oscillation at $37^{\circ} \mathrm{C}$ for $30 \mathrm{~min}$. After centrifugation at $1000 \mathrm{rpm}$ for 5 min, the supernatant was removed. DMEM containing 10\% FBS was added to terminate the reaction. After filtering with a $100-\mu \mathrm{m}$ mesh cell sieve, ASMCs were inoculated into a $50-\mathrm{mL}$ bottle for culture at $37^{\circ} \mathrm{C}$, followed by purification according to the faster adherence characteristic of fibroblasts. After the bottle bottom was completely covered with cells, the cells were passaged (1:2) and 3rd-generation cells were collected for subsequent use.

\section{Detection of F-actin and $\alpha$-tubulin by immunofluorescence staining}

ASMCs were fixed with 4\% paraformaldehyde at room temperature for $20 \mathrm{~min}$, followed by treatment with $0.1 \%$ Triton X-100 for $10 \mathrm{~min}$. In dark conditions, $5 \mu \mathrm{g} / \mathrm{mL}$ phalloidin-FITC was added for staining at $37^{\circ} \mathrm{C}$ for $1 \mathrm{~h}$. After sealing with $50 \%$ glycerol, slices were observed by fluorescence microscopy for F-actin and $\alpha$-tubulin detection.

\section{Detection of RhoA and ROCK mRNA expressions by real-time qPCR}

Standard curves of the target RhoA and ROCK genes, and the housekeeping gene, GAPDH, were constructed using the SYBR Green method. According to the standard curve, the three genes were quantitated. The relative expression level of target gene mRNA was calculated as follows: copy number of target gene/copy number of GAPDH gene. The primers used were as follows: RhoA primer (upstream, 5'-AACTGGTGATTGTTGGTG-3' and down- 
stream, 5'-CTGCCACATAGTTTTCAA-3'), amplified fragment length, $114 \mathrm{bp}$; ROCK primer (upstream, 5'-GAGTCTGCTGGATGGCTT-3' and downstream, 5'-TCTTCCGCTTTCATCTG T-3'), amplified fragment length, $141 \mathrm{bp}$; GAPDH primer (upstream, 5'-GCACCGTCAAGGC TGAGAAC-3' and downstream, 5'-ATGGTGGTGAAGACGCCAGT-3'), amplified fragment length, $142 \mathrm{bp}$.

\section{Detection of $\alpha$-tubulin, RhoA, and ROCK protein expressions by Western blot analysis}

The bicinchoninic acid assay was used for protein quantification. After 10\% sodium dodecyl sulfate polyacrylamide gel electrophoresis was performed (stacking gel voltage, 80 $\mathrm{V}$; separating gel voltage, $120 \mathrm{~V}$ ), the gel was transferred to nitrocellulose membranes at a constant current of $280 \mathrm{~mA}$ for $50 \mathrm{~min}$. Rabbit anti-rat antibody (1:400) and alkaline phosphatase-labeled goat anti-rabbit secondary antibody $(1: 2000)$ were used for coloration. The gray value of each gel band was recorded and analyzed quantitatively using the FluorChem V2.0 software (USA). The protein content was calculated as follows: gray value of protein/gray value of $\beta$-actin in the same sample.

\section{RESULTS}

\section{Morphological observations of ASMCs}

ASMCs were observed under an inverted microscope. Before adherence, ASMCs were found to be round or oval, and opaque with high-density cytoplasm. After 1 day of culture, most cells adhered to the wall and began to stretch. After 3 days of culture, the adherent cells were fully stretched, presenting fusiform or polygon shapes. There were 1-2 nuclei in the center of cells, with one or more foot processes. There was a fascicular arrangement of cells in one region, which displayed a "peak-like" morphological feature. After 7-9 days of culture, the bottom of the bottle was completely covered by cells. The time to complete covering of cells on the bottle bottom was inversely proportional to cell viability and seeding density; time decreased with increasing cell viability and inoculation density (Figure 1).

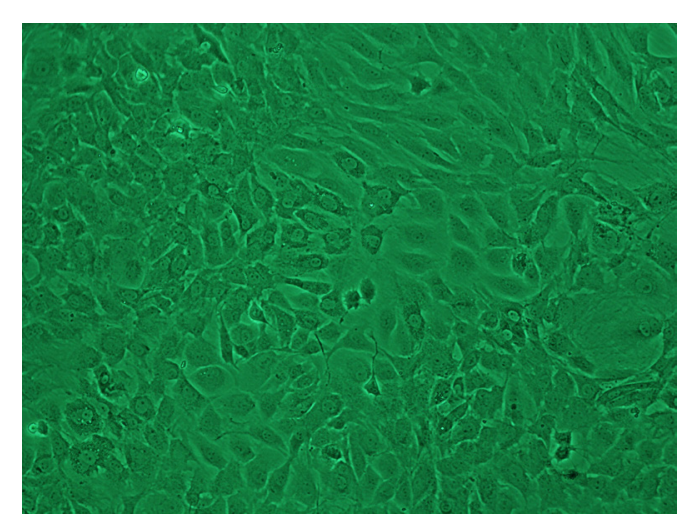

Figure 1. Morphological observation of airway smooth muscle cells (100X). 


\section{Identification of ASMCs by immunofluorescent staining}

$\alpha$-SMA immunofluorescent staining was used for identification of ASMCs. Under a fluorescence microscope with a wavelength of $488 \mathrm{~nm}$, FITC-labeled $\alpha$-SMA appeared green, and they were uniformly distributed in the cytoplasm for the most part. DAPI-labeled nuclei exhibited blue fluorescence at a wavelength of $356 \mathrm{~nm}$ (Figure 2).

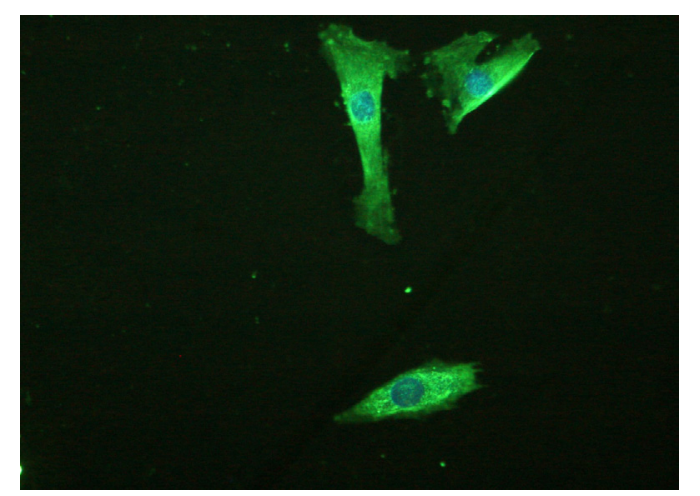

Figure 2. Identification of airway smooth muscle cells by $\alpha$-SMA immunofluorescent staining (400X).

\section{Expression of F-actin in ASMCs}

Phalloidin could specifically bind intracellular F-actin and exhibited green fluorescence. Under the fluorescence microscope, few short and fine stress fibers were observed in ASMCs in the control group. Cells appeared stretched in asthmatic groups, and showed a relatively higher number of stress fibers. The average gray values of F-actin in ASMCs of asthmatic groups were all higher than those of the control group. The average gray value of F-actin in ASMCs of the intervention group was significantly lower than that of the 8-week asthmatic group (Table 1; Figure 3).

Table 1. Comparisons of F-actin gray value and $\alpha$-tubulin protein among the different groups (means $\pm \mathrm{SD})$.

\begin{tabular}{lcc}
\hline Group & F-actin gray value & $\alpha$-tubulin protein \\
\hline Control group & $75.64 \pm 8.69$ & $0.1187 \pm 0.0015$ \\
2-week asthmatic group & $91.05 \pm 10.86^{*}$ & $0.1489 \pm 0.0019$ \\
4-week asthmatic group & $126.84 \pm 14.54^{* *}$ & $0.1591 \pm 0.0023^{*}$ \\
6-week asthmatic group & $171.07 \pm 16.81^{* *}$ & $0.1642 \pm 0.0024^{*}$ \\
8-week asthmatic group & $247.92 \pm 25.58^{* *}$ & $0.1695 \pm 0.0027^{* *}$ \\
Intervention group & $136.02 \pm 13.84^{\Delta \Delta}$ & $0.1275 \pm 0.0024^{\Delta}$ \\
\hline
\end{tabular}

$* \mathrm{P}<0.05$ compared to control group; $* * \mathrm{P}<0.01$ compared to control group; ${ }^{\mathrm{P}}<0.05$ compared to asthmatic groups; ${ }^{\Delta \Delta} \mathrm{P}<0.01$ compared to 8 -week asthmatic group.

\section{Distribution and morphology of $\alpha$-tubulin in ASMCs}

$\alpha$-tubulin exhibited green fluorescence after staining. The microtubule structure in the cytoplasm showed a high-density mesh-like and radiation-like distribution from the nuclear 
center to the periphery. The fluorescence signal intensity and density were significantly higher in all asthmatic groups relative to the control group. The fluorescence signal was significantly weakened in the intervention group, which showed a more pronounced mesh structure and more fragments (Figure 4).
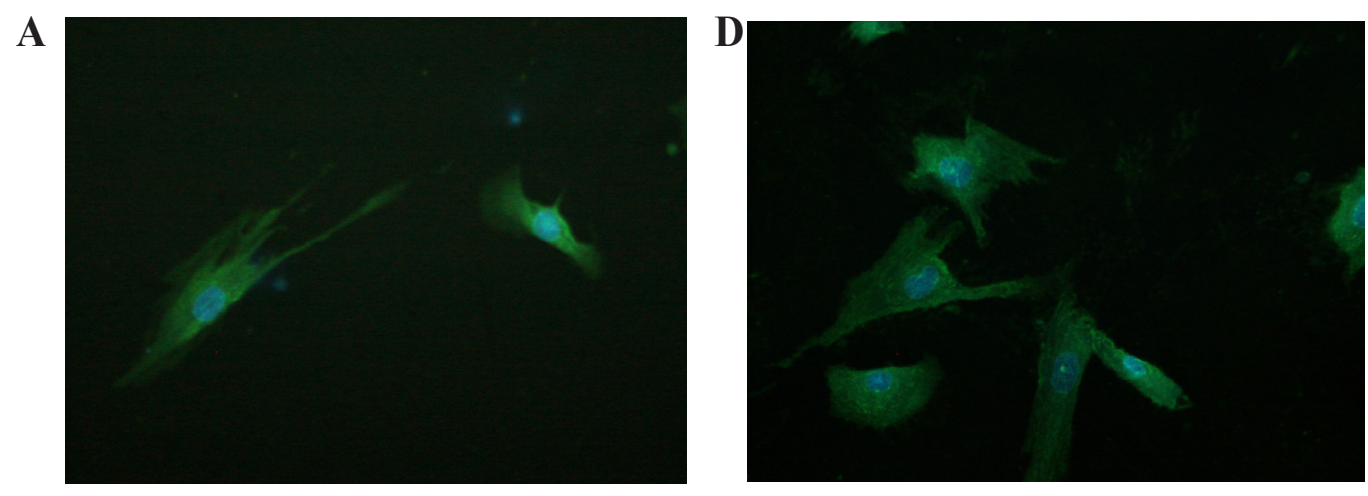

B
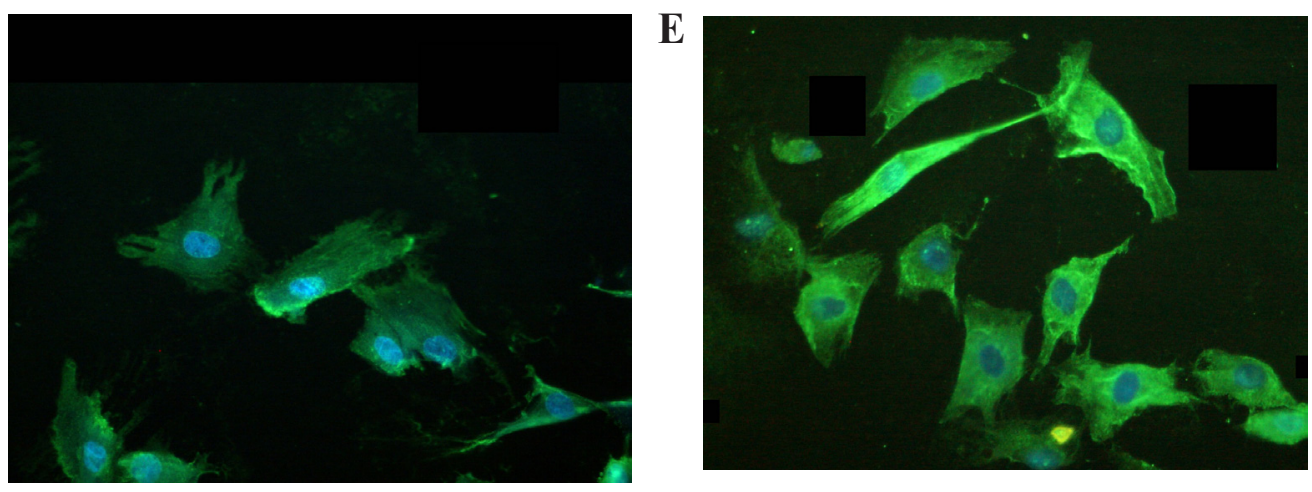

$\mathrm{C}$
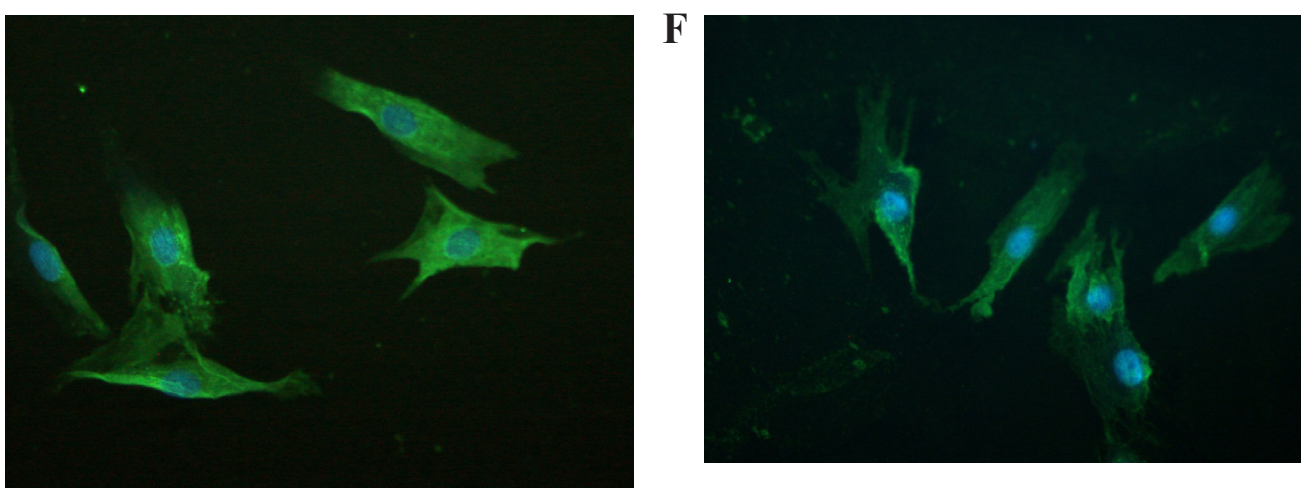

Figure 3. Immunofluorescence staining of F-actin in airway smooth muscle cells. A. Control group; B. 2-week asthmatic group; C. 4-week asthmatic group; D. 6-week asthmatic group; E. 8-week asthmatic group; F. intervention group. 
A

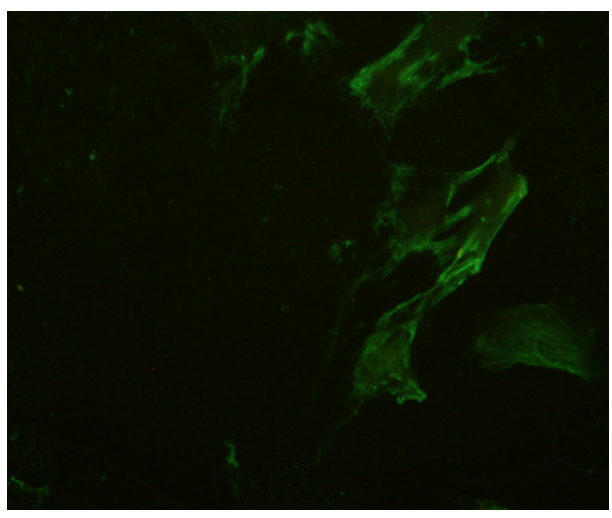

B

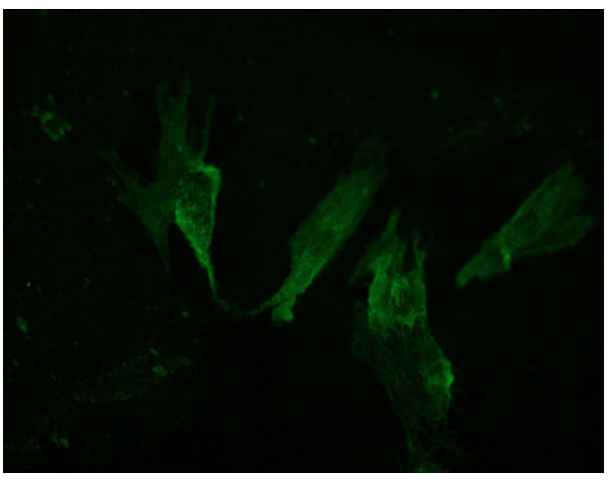

C

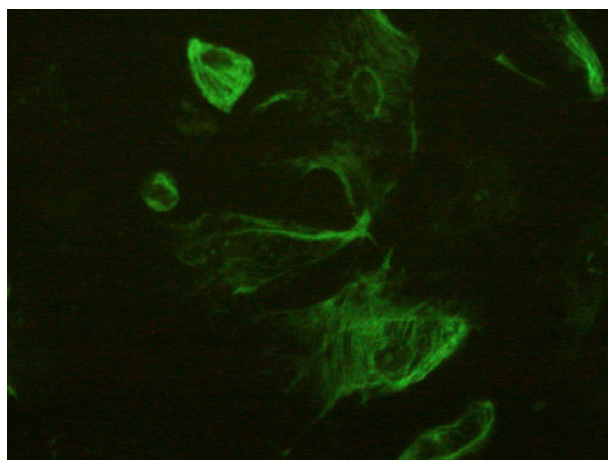

D

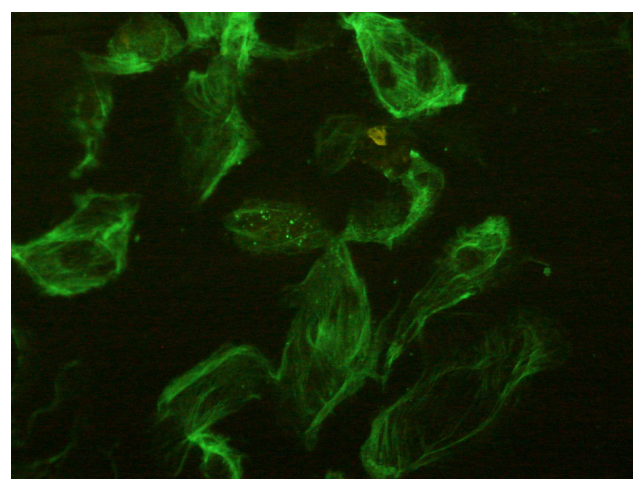

$\mathbf{E}$

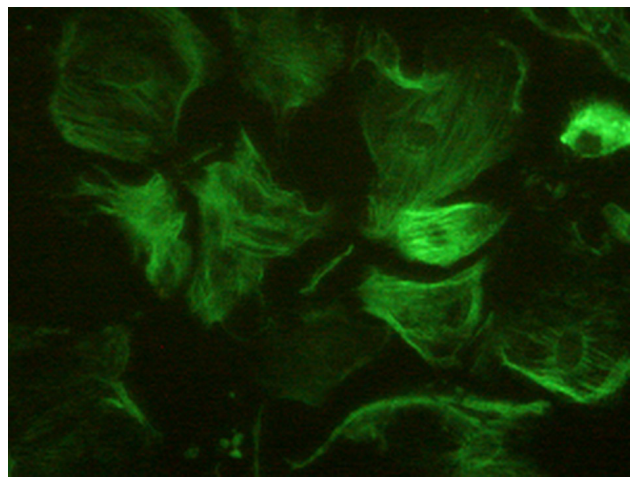

$\mathbf{F}$

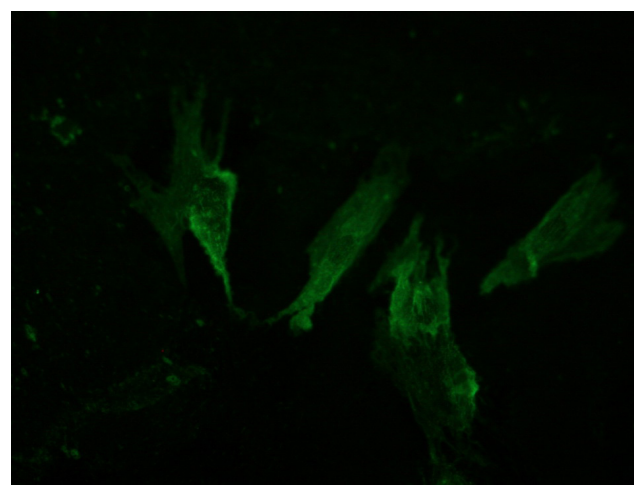

Figure 4. Immunofluorescence staining of $\alpha$-tubulin in airway smooth muscle cells. A. Control group; B. 2-week asthmatic group; C. 4-week asthmatic group; D. 6-week asthmatic group; E. 8-week asthmatic group; F. intervention group. 


\section{Expression of the $\alpha$-tubulin protein in ASMCs}

Expressions of the $\alpha$-tubulin protein in ASMCs were significantly higher in all asthmatic groups than in the control group, and this difference increased with the extension of excitation time. The difference was most apparent in the 8-week asthmatic group. The expression level of $\alpha$-tubulin was significantly reduced in the intervention group (Table 1; Figure 5).

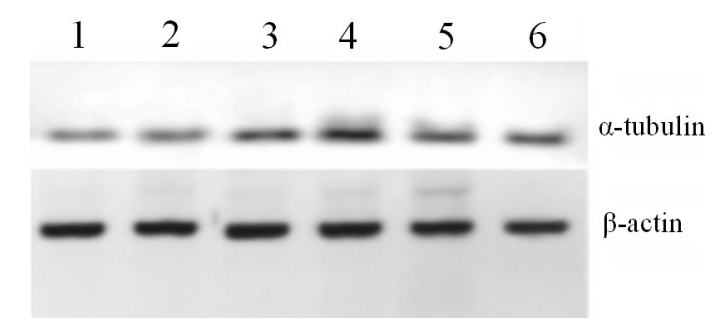

Figure 5. Expression of $\alpha$-tubulin protein in airway smooth muscle cells. Lane $1=$ control group; lane $2=2$-week asthmatic group; lane $3=4$-week asthmatic group; lane $4=6$-week asthmatic group; lane $5=8$-week asthmatic group; lane $6=$ intervention group.

\section{Expressions of RhoA and ROCK mRNA and proteins in ASMCs}

Expressions of RhoA and ROCK mRNA and proteins in ASMCs were significantly higher in all asthmatic groups relative to those of the control group, and the difference increased with the extension of excitation time. Once again, this result was more significant in the 8-week asthmatic group (Table 2; Figure 6).

Table 2. Expressions of RhoA and ROCK mRNA and protein in airway smooth muscle cells (means $\pm \mathrm{SD}$ ).

\begin{tabular}{lllll}
\hline Group & \multicolumn{1}{c}{ RhoA mRNA } & ROCK mRNA & RhoA protein & ROCK protein \\
\hline Control group & $0.0241 \pm 0.0002$ & $0.0197 \pm 0.0015$ & $0.1687 \pm 0.0015$ & $0.1388 \pm 0.0012$ \\
2-week asthmatic group & $0.0382 \pm 0.0003^{*}$ & $0.0258 \pm 0.0019^{*}$ & $0.1774 \pm 0.0018$ & $0.1679 \pm 0.0015^{*}$ \\
4-week asthmatic group & $0.0401 \pm 0.0002^{*}$ & $0.0267 \pm 0.0023^{*}$ & $0.1798 \pm 0.0022$ & $0.1741 \pm 0.0019^{*}$ \\
6-week asthmatic group & $0.0462 \pm 0.0002^{* *}$ & $0.0288 \pm 0.0024^{* *}$ & $0.1842 \pm 0.0024^{*}$ & $0.1796 \pm 0.0019$ \\
8-week asthmatic group & $0.0527 \pm 0.0004^{* *}$ & $0.0299 \pm 0.0027^{* *}$ & $0.1903 \pm 0.0022^{* *}$ & $0.1842 \pm 0.0021^{* *}$ \\
\hline
\end{tabular}

$* \mathrm{P}<0.05$ compared to control group; $* * \mathrm{P}<0.01$ compared to control group.

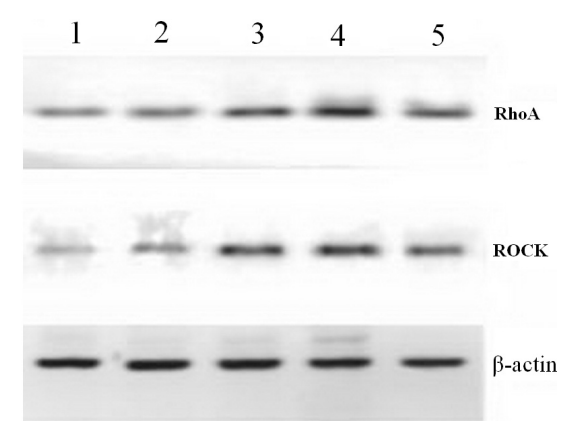

Figure 6. Expressions of RhoA and ROCK mRNA and protein in airway smooth muscle cells. Lane $1=$ control group; lane 2 = 2-week asthmatic group; lane 3=4-week asthmatic group; lane 4=6-week asthmatic group; lane $5=8$-week asthmatic group. 


\section{DISCUSSION}

The cytoskeleton is a type of fibrous protein matrix of eukaryotic cells, and presents mesh, fascicular, and banding shapes. It is composed of microfilaments, microtubules, and intermediate filaments, which are highly coordinated with the cell nucleus, plasma membrane, and cell organs, and constitute the cellular skeleton and motion coordination system. The cytoskeleton can maintain the cell morphology and exercise motor functions, and plays an important role in signal transmission (Paavilainen et al., 2004).

Microfilaments are polar fine fibers with $7 \mathrm{~mm}$ in diameters, which are mainly composed of actins, and they determine the intracellular spiral-shape. Microfilaments are the central structure forming stress fibers, muscle fibers, and the crypt-villus axis of the small intestine. Actin is the most abundant protein in most eukaryotic cells, and has been highly conserved in evolution, showing little variation among species. The actin family includes Gactin and F-actin, and the cytoskeleton is mainly composed of F-actin. Actin cytoskeletons are related to many cellular functions. They participate in muscle formation and cell movement. In addition, actin cytoskeletons can control transmission of mRNA-related information and transportation of intracellular vesicles and receptors. In local cellular membranes, actin cytoskeletons aggregate to form fascicular stress fibers. One end of the stress fiber accepts the stimulatory signal of the extracellular matrix, while the other end is connected to cytoplasmic organelles to control adhesion and signal transmission between the cell and the extracellular matrix. At the same time, the functions of microfilaments are regulated by a signal transduction system. Actin is generally considered to be the first target protein for the initiation of extracellular signals (e.g., cytokines) and in the regulation of intracellular signals (Plastino and Sykes, 2005; Gouëffic et al., 2006; Nincheri et al., 2009; Abounit and Zurzolo, 2012; Martin et al., 2012; Karvonen et al., 2012). FITC-labeled phalloidin can specifically bind F-actin, but not G-actin, enabling observation of structural and distributional changes of F-actin.

Results of the present study revealed that in the control group, a small amount of short and fine stress fibers, without filamentary pseudopod formations, were observed under a fluorescence microscope. In contrast, in asthmatic groups, cells were visibly stretched, with increased stress fibers and obvious pseudopods. This indicates actin cytoskeleton reconstruction in ASMCs in rats with airway remodeling in asthma.

The microtubule is a hollow cylindrical tubular fiber with a diameter of $25 \mathrm{~nm}$ and is mainly composed of $\alpha$ - and $\beta$-tubulin ( $\alpha$ - and $\beta$-dimer). Furthermore, it is a GTP-binding protein. Microtubules have obvious amino acid sequence homology with $G$ proteins of the signal transduction system, and have similar functional characteristics to G proteins (Pao et al., 2009; Lee et al., 2013). They are involved in cell movement and cytoplasmic transportation, as well as chromosome movement and mitosis (Sahab et al., 2011; Sharp et al., 2011; Hinrichs et al., 2012). Therefore, microtubules are associated with cell proliferation. However, investigations of the mechanism of airway remodeling in asthma in view of the microtubule cytoskeleton are rare. In this study, ASMCs in rats with airway remodeling in asthma were cultured, and immunofluorescence staining and Western blot analysis were used to detect the expression of $\alpha$-tubulin. Results revealed that $\alpha$-tubulin expression levels were significantly higher in asthmatic groups than in the control group. This suggests that changes of the microtubule cytoskeleton besides microfilament reconstruction persist in rats with airway remodeling in asthma.

RhoA is an important molecule for mediating cell signal transduction. Similar to $G$ proteins, GTP enzymes of RhoA can shift between GTP binding states (activation) and GDP 
binding states (inactivated). It triggers the downstream kinase cascade reaction and exerts several biological effects (Ding et al., 2011). RhoA is also the primary pathway mediating stress fiber formation. It combines with downstream target molecules such as ROCK to directly modulate contractile proteins to participate in actin cytoskeleton reconstruction. In addition, it can change the activity of serum response factors and regulate gene expressions of different contractile proteins for long-term adjustments of the actin cytoskeleton. RhoA is also known as a regulator of heterogeneity of the actin cytoskeleton and cellular morphology (Thapar et al., 2002; dos Remedios et al., 2003; Mohseni and Chishti, 2009). Recent studies have demonstrated that the RhoA/ROCK signaling pathway is involved in many pathophysiological processes of asthma, including formation of asthmatic airway hyper-responsiveness, chemotactic aggregation of eosinophil to the airway, and airway remodeling (Bamburg and Wiggan, 2002; Takeda et al., 2006; Kume, 2008; Li et al., 2008; Goto et al., 2010).

In the present study, qPCR and Western blot methods were used to detect mRNA and protein expressions of RhoA and ROCK in rats with airway remodeling in asthma. Results showed that mRNA and protein expressions of RhoA and ROCK were significantly upregulated in all asthmatic groups relative to the control group. After treatment with a ROCK-specific inhibitor, the expression levels of F-actin and $\alpha$-tubulin decreased, suggesting that the RhoA/ ROCK signal may induce changes in the microfilament and microtubule cytoskeleton.

\section{CONCLUSIONS}

The cytoskeleton and RhoA/ROCK may signal transduction pathways in which external stimulations induce biological functions of ASMCs. They can be present at both the front and back ends of information transmission chains, or exist independently. These pathways are connected, and they cooperate and restrict one another, thereby forming a crisscross information transmission network. Whether other pathways are also involved requires further study. In-depth investigations of such mechanisms can broaden the prospects for studying the pathogenesis of airway remodeling as well as for development of novel strategies for its clinical prevention and treatment.

\section{REFERENCES}

Abounit S and Zurzolo C (2012). Wiring through tunneling nanotubes-from electrical signals to organelle transfer. J. Cell Sci. 125: 1089-1098.

Bamburg JR and Wiggan OP (2002). ADF/cofilin and actin dynamics in disease. Trends Cell Biol. 12: 598-605.

Ding F, Yin Z and Wang HR (2011). Ubiquitination in Rho signaling. Curr. Top. Med. Chem. 11: 2879-2887.

dos Remedios CG, Chhabra D, Kekic M, Dedova IV, et al. (2003). Actin binding proteins: regulation of cytoskeletal microfilaments. Physiol. Rev. 83: 433-473.

Fukata Y, Amano M and Kaibuchi K (2001). Rho-Rho-kinase pathway in smooth muscle contraction and cytoskeletal reorganization of non-muscle cells. Trends Pharmacol. Sci. 22: 32-39.

Goto K, Chiba Y, Sakai H and Misawa M (2010). Mechanism of inhibitory effect of prednisolone on RhoA upregulation in human bronchial smooth muscle cells. Biol. Pharm. Bull. 33: 710-713.

Gouëffic Y, Guilluy C, Guérin P, Patra P, et al. (2006). Hyaluronan induces vascular smooth muscle cell migration through RHAMM-mediated PI3K-dependent Rac activation. Cardiovasc. Res. 72: 339-348.

Hinrichs MH, Jalal A, Brenner B, Mandelkow E, et al. (2012). Tau protein diffuses along the microtubule lattice. J. Biol. Chem. 287: 38559-38568.

Karvonen HM, Lehtonen ST, Sormunen RT, Harju TH, et al. (2012). Myofibroblasts in interstitial lung diseases show diverse electron microscopic and invasive features. Lab. Invest. 92: 1270-1284. 
Kume H (2008). RhoA/Rho-kinase as a therapeutic target in asthma. Curr. Med. Chem. 15: 2876-2885.

Lee MN, Koh A, Park D, Jang JH, et al. (2013). Deacetylated $\alpha \beta$-tubulin acts as a positive regulator of Rheb GTPase through increasing its GTP-loading. Cell Signal. 25: 539-551.

Li ZX, Luo YL, Xu J, Ren DQ, et al. (2008). Rho-kinase signaling pathway participates in endothelin-1-induced human airway smooth muscle cell migration and cytoskeletal reorganization. Nan. Fang Yi Ke. Da Xue Xue Bao 28: 10311034.

Martin E, Dahan D, Cardouat G, Gillibert-Duplantier J, et al. (2012). Involvement of TRPV1 and TRPV4 channels in migration of rat pulmonary arterial smooth muscle cells. Pflugers Arch. 464: 261-272.

Mohseni M and Chishti AH (2009). Regulatory models of RhoA suppression by dematin, a cytoskeletal adaptor protein. Cell Adh. Migr. 3: 191-194.

Nincheri P, Luciani P, Squecco R, Donati C, et al. (2009). Sphingosine 1-phosphate induces differentiation of adipose tissue-derived mesenchymal stem cells towards smooth muscle cells. Cell. Mol. Life Sci. 66: 1741-1754.

Paavilainen VO, Bertling E, Falck S and Lappalainen P (2004). Regulation of cytoskeletal dynamics by actin-monomerbinding proteins. Trends Cell Biol. 14: 386-394.

Pao CS, Barker BL and Benovic JL (2009). Role of the amino terminus of G protein-coupled receptor kinase 2 in receptor phosphorylation. Biochemistry 48: 7325-7333.

Plastino J and Sykes C (2005). The actin slingshot. Curr. Opin. Cell Biol. 17: 62-66.

Sahab ZJ, Hall MD, Me SY, Dakshanamurthy S, et al. (2011). Tumor suppressor RARRES1 interacts with cytoplasmic carboxypeptidase AGBL2 to regulate the $\alpha$-tubulin tyrosination cycle. Cancer Res. 71: 1219-1228.

Sharp JA, Plant JJ, Ohsumi TK, Borowsky M, et al. (2011). Functional analysis of the microtubule-interacting transcriptome. Mol. Biol. Cell 22: 4312-4323.

Stamenović D (2008). Cytoskeletal mechanics in airway smooth muscle cells. Respir. Physiol. Neurobiol. 163: 25-32.

Takeda N, Kondo M, Ito S, Ito Y, et al. (2006). Role of RhoA inactivation in reduced cell proliferation of human airway smooth muscle by simvastatin. Am. J. Respir. Cell Mol. Biol. 35: 722-729.

Thapar R, Karnoub AE and Campbell SL (2002). Structural and biophysical insights into the role of the insert region in Rac1 function. Biochemistry 41: 3875-3883. 